\title{
Nuclear Spin Switch in Semiconductor Quantum Dots
}

\author{
A. I. Tartakovskii, ${ }^{1}$ T. Wright, ${ }^{1}$ A. Russell, ${ }^{2}$ V. I. Fal'ko, ${ }^{2}$ A. B. Van'kov, ${ }^{1}$ J. Skiba-Szymanska, ${ }^{1}$ I. Drouzas, ${ }^{1}$ \\ R. S. Kolodka, ${ }^{1}$ M. S. Skolnick, ${ }^{1}$ P. W. Fry, ${ }^{3}$ A. Tahraoui, ${ }^{3}$ H.-Y. Liu, ${ }^{3}$ and M. Hopkinson ${ }^{3}$ \\ ${ }^{1}$ Department of Physics and Astronomy, University of Sheffield, S3 7RH, United Kingdom \\ ${ }^{2}$ Department of Physics, University of Lancaster, Lancaster LA1 4YB, United Kingdom \\ ${ }^{3}$ Department of Electronic and Electrical Engineering, University of Sheffield, Sheffield S1 3JD, United Kingdom
}

(Received 12 September 2006; published 12 January 2007)

\begin{abstract}
We show that by illuminating an InGaAs/GaAs self-assembled quantum dot with circularly polarized light, the nuclei of atoms constituting the dot can be driven into a bistable regime, in which either a thresholdlike enhancement or reduction of the local nuclear field by up to $3 \mathrm{~T}$ can be generated by varying the pumping intensity. The excitation power threshold for such a nuclear spin "switch" is found to depend on both the external magnetic and electric fields. The switch is shown to arise from the strong feedback of the nuclear spin polarization on the dynamics of the spin transfer from electrons to the nuclei of the dot.
\end{abstract}

DOI: 10.1103/PhysRevLett.98.026806

PACS numbers: 73.21.La, 72.25.Fe

The hyperfine interaction in solids [1] arises from the coupling between the magnetic dipole moments of nuclear and electron spins. In quantum dots (QDs), the electron coupling to the underlying nuclei is stronger than in a bulk material due to the electron confinement [2-9]. This leads to the enhanced contribution of the hyperfine interaction to the electron spin decoherence [3] and relaxation $[4,8,9]$ at low temperatures. In this Letter, we show that in optically pumped individual self-assembled InGaAs/GaAs dots the enhanced coupling between the electron and nuclear spins gives rise to a pronounced bistability of the nuclear spin polarization.

In our photoluminescence (PL) experiments, spinpolarized electrons are introduced one by one into an individual InGaAs dot at a rate $w_{x}$ by the circularly polarized optical excitation of electron-hole pairs $120 \mathrm{meV}$ above the lowest QD energy states [see Fig. 1(a)]. Both bright and dark excitons can form in the dot ground state due to hole spin-flip during its energy relaxation. The former will quickly recombine radiatively with a rate $w_{\text {rec }} \sim 10^{9} \mathrm{sec}^{-1}$, whereas the dark exciton can recombine with simultaneous spin transfer to one of $N$ nuclei in a dot $\left(N \sim 10^{4}\right)$ via a spin "flip-flop" process [as in Fig. 1(b)]. The rate of this process is $w_{\text {rec }} N p_{\text {hf }}[8,10]$, where $p_{\text {hf }}$ is the probability of a spin "flip-flop" process and is given by:

$$
p_{\mathrm{hf}}=\left|h_{\mathrm{hf}}\right|^{2} /\left(E_{\mathrm{eZ}}^{2}+\frac{1}{4} \gamma^{2}\right) \text {. }
$$

Here $\gamma$ is the electron lifetime broadening, $h_{\mathrm{hf}}$ is the strength of the hyperfine interaction of the electron with a single nucleus, and $E_{\mathrm{eZ}}$ is the electron Zeeman splitting. $E_{\mathrm{eZ}}$ is dependent on both external and effective nuclear (Overhauser) magnetic fields, where the latter, $B_{N}$, is proportional to the degree of nuclear polarization, $S$. This provides a feedback mechanism between the spin transfer rate, $w_{s}$, and nuclear polarization in the dot leading to the enhancement of $w_{s}$ in the case when $B_{N}$ acts to reduce the electron Zeeman splitting. The feedback gives rise to bi- stability in the nuclear polarization and thresholdlike transitions between the spin states of $10^{4}$ nuclei (referred to below as a nuclear spin "switch") leading to abrupt changes of $B_{N}$ by up to $3 \mathrm{~T}$.

We observe the nuclear spin switch in several different structures containing self-assembled InGaAs QDs with $\sim 3 \times 20 \times 20 \mathrm{~nm}$ size. Below, we present results obtained at a temperature of $15 \mathrm{~K}$ for two GaAs/AlGaAs based Schottky diodes [11], where the dots are grown in the intrinsic region of the device. Figure 1(c) shows timeaveraged (60 s) PL spectra recorded for a neutral exciton

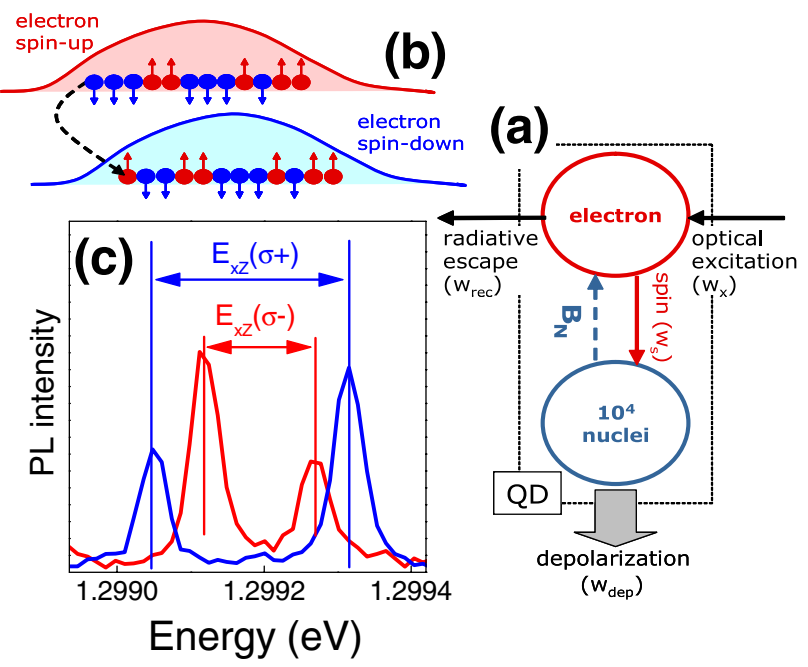

FIG. 1 (color online). (a) The steps involved in nuclear polarization of a quantum dot (see text for detail). (b) Schematic diagram of the electron-nuclear spin "flip-flop," in which the electron confined on the dot flips its spin simultaneously flipping the spin of a single nucleus. (c) $X^{0}$ PL spectra recorded for an individual InGaAs QD in an external magnetic field $B=2 \mathrm{~T}$ at $T=15 \mathrm{~K}$. The spectrum excited with $\sigma^{+}\left(\sigma^{-}\right)$light resonant with the wetting layer is plotted in blue (red). The horizontal arrows show the corresponding exciton Zeeman splittings. 
in a single QD in an external magnetic field of $2 \mathrm{~T}$. Circularly polarized laser excitation at $1.425 \mathrm{eV}$ is employed and unpolarized PL from the dot is detected using a double spectrometer and a CCD. For each excitation polarization a spectrum consisting of an exciton Zeeman doublet is measured with the high (low) energy component dominating when $\sigma^{+}\left(\sigma^{-}\right)$polarization is used. A strong dependence of the exciton Zeeman splitting $\left(E_{\mathrm{xZ}}\right)$ on the polarization of the excitation is observed in Fig. 1(c): $E_{\mathrm{xZ}}\left(\sigma^{+}\right)=260 \mu \mathrm{eV}$ and $E_{\mathrm{xZ}}\left(\sigma^{-}\right)=150 \mu \mathrm{eV}$. Such a dependence is a signature of dynamic nuclear polarization [2,6,7], which gives rise to the Overhauser field $B_{N}$ aligned parallel (antiparallel) to $B$ for $\sigma^{+}\left(\sigma^{-}\right)$excitation.

The dependence of exciton PL at $B=2.5 \mathrm{~T}$ on the power, $P \propto w_{x}$, of $\sigma^{-}$excitation is shown in the gray-scale plot in Fig. 2(a) [12]. At low $P$ the Zeeman splitting $E_{\mathrm{xZ}}=$ $310 \mu \mathrm{eV}$. As the power is increased, a thresholdlike decrease of $E_{\mathrm{xZ}}$ to $225 \mu \mathrm{eV}$ is observed at $P=P_{\text {up }}$ indicating the sudden appearance of a large nuclear field. Figure 2(b) shows the power dependence of $E_{\mathrm{xZ}}$ measured at $B=2 \mathrm{~T}$ for both circular polarizations of incident light. For $\sigma^{-}$excitation, $E_{\mathrm{xZ}}$ decreases below the threshold followed by a weak power dependence above the threshold [13]. The $\sigma^{-}$behavior contrasts to the weak monotonic

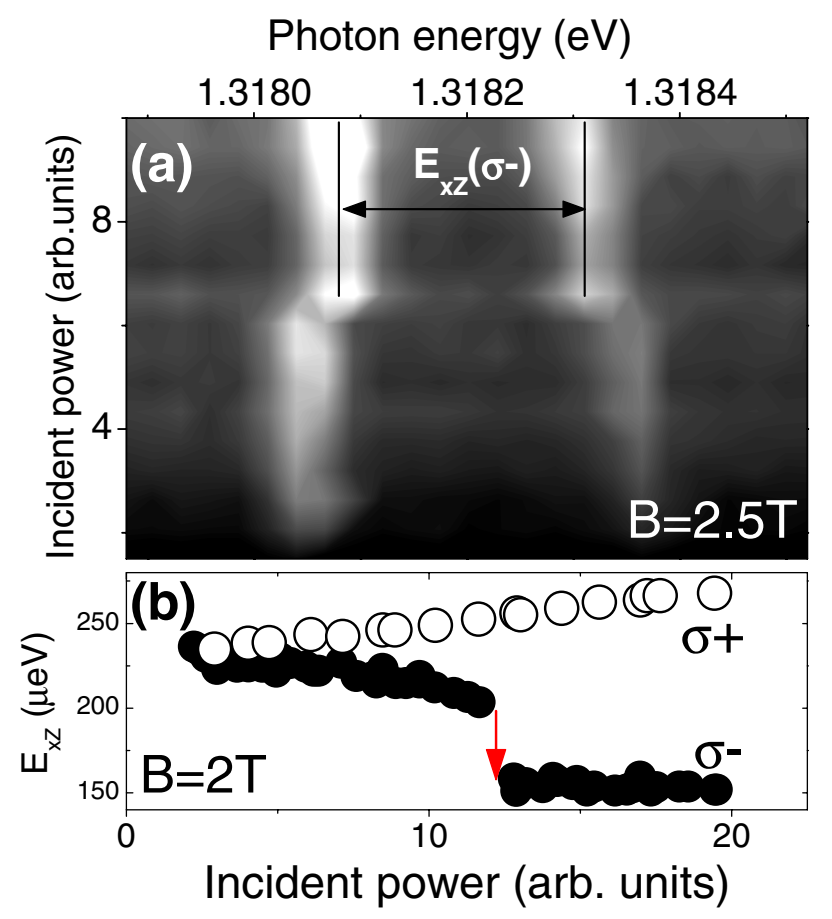

FIG. 2 (color online). (a) Gray-scale plot showing exciton PL spectra recorded for an individual InGaAs dot. The spectra are recorded at $B=2.5 \mathrm{~T}$ using unpolarized detection and $\sigma^{-}$ excitation into the wetting layer. The spectra are displaced along the vertical axis according to the excitation power at which they are measured. (b) $E_{\mathrm{xZ}}$ power dependences measured at $B=2 \mathrm{~T}$ for $\sigma^{+}$and $\sigma^{-}$excitation polarizations. increase of $E_{\mathrm{xZ}}$ seen for $\sigma^{+}$excitation over the whole range of powers.

The variation of the Zeeman splitting in Fig. 2 reflects the change in the nuclear field $B_{N}: E_{\mathrm{xZ}}\left(\sigma^{ \pm}\right)=$ $\left|g_{e}+g_{h}\right| \mu_{B} B \pm\left|g_{e}\right| \mu_{B} B_{N}\left(\sigma^{ \pm}\right)$[where $g_{e}\left(g_{h}\right)$ is the electron (hole) $g$ factor [14], $\mu_{B}$ is the Bohr magneton]. $B_{N}$ in its turn depends on the external field $B$. The triangles in Fig. 3 show the difference between $E_{\mathrm{xz}}\left(\sigma^{-}\right)$at low and high powers (squares and circles in Fig. 3, respectively), $\Delta E_{N}=\left|g_{e}\right| \mu_{B} B_{N}\left(\sigma^{-}\right)$, as a function of $B . \Delta E_{N}$ increases linearly with $B$ at low fields and then saturates at $B \approx$ 2.5-3 T. For a typical $\operatorname{In}_{0.3} \mathrm{Ga}_{0.2}$ As dot, the maximum $\Delta E_{N}$ in Fig. 3 corresponds to a nuclear polarization of $40 \%$ [7]. The inset in Fig. 3 shows that the threshold power for the switch also increases nearly linearly with $B$. No switch could be observed at $B>3 \mathrm{~T}$ in the range of powers employed in our studies.

For $B<3 \mathrm{~T}$, when the excitation power was gradually reduced from powers above the switch, $E_{\mathrm{xZ}}$ was found to vary weakly with power until another threshold was reached, where the magnitude of the exciton Zeeman splitting abruptly increased (at $P=P_{\text {down }}$ ), as shown in Fig. 4. This increase of $E_{\mathrm{xZ}}$ corresponds to depolarization of the nuclei and hence reduction of $B_{N}$. The observed hysteresis of nuclear polarization shows that two significantly different and stable nuclear spin configurations can exist for the same external parameters such as magnetic field and excitation power. We find that high nuclear polarization persists at low excitation powers for more than $15 \mathrm{~min}$, this time most likely being determined by the stability of the experimental setup.

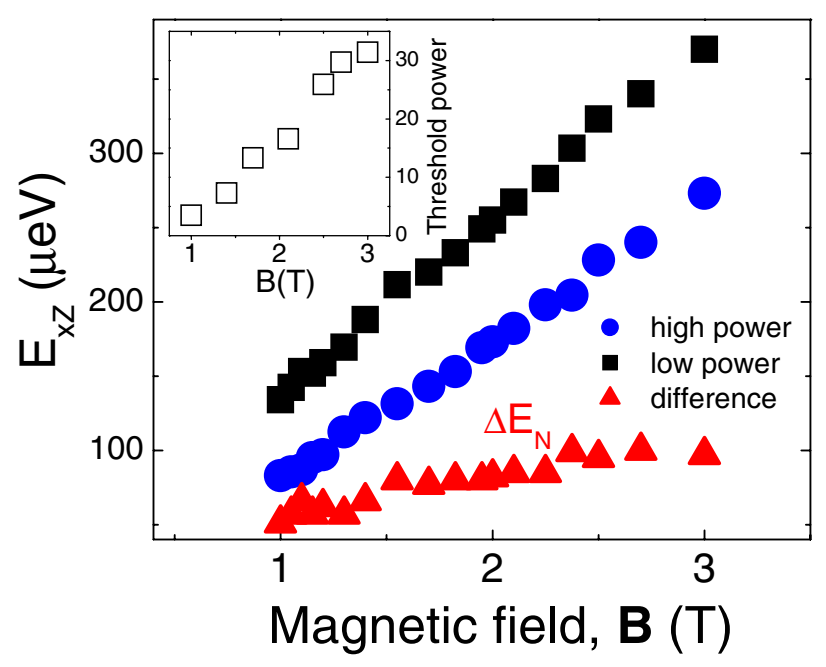

FIG. 3 (color online). Dependence of the QD exciton Zeeman splitting $E_{\mathrm{xZ}}\left(\sigma^{-}\right)$on the external magnetic field. Squares and circles show high and low power data, respectively, and triangles show their difference, $\Delta E_{N}$. For all $B$ shown in the figure the nuclear switch threshold was observed with the threshold power shown in the inset as a function of $B$. 


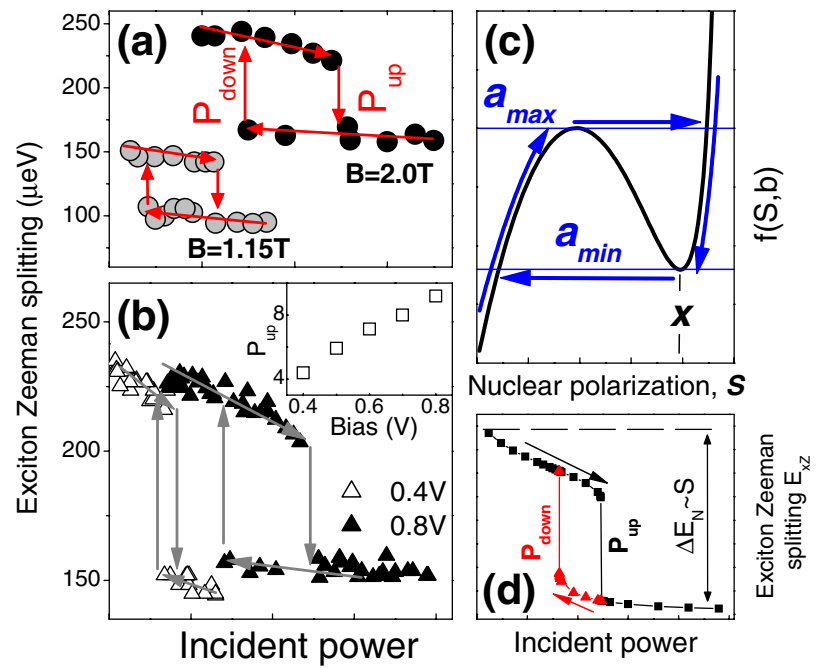

FIG. 4 (color online). (a) Power dependence of $E_{\mathrm{xZ}}\left(\sigma^{-}\right)$measured at $B=2 \mathrm{~T}$ and $1.15 \mathrm{~T}$. The arrows show the direction in which the hysteresis loop is measured with two thresholds $P_{\text {up }}$ and $P_{\text {down }}$. (b) $E_{\mathrm{xZ}}\left(\sigma^{-}\right)$power dependence measured at $B=$ $2.0 \mathrm{~T}$. The two hysteresis loops are measured at 0.4 and $0.8 \mathrm{~V}$ applied bias. The inset shows the $P_{\text {up }}$ dependence on the reverse bias applied to the diode. (c) The full line shows the function $f(S, b)$ from Eq. (2). Arrows show how the hysteresis loop is formed when the parameter $a\left(\propto w_{x}\right)$ is varied for a fixed $b$. (d) Hysteresis loop of the exciton Zeeman splitting as a function of incident power calculated using Eq. (2) for $x=0.7$ and $\theta=0.1$.

We also show in Figs. 4(a) and 4(b) that the size of the hysteresis loop depends on the external magnetic or electric fields [the electric field is given by $F=\left(V_{\text {rev }}+\right.$ $0.7 V) / d$, where $V_{\text {rev }}$ is the applied reverse bias and $d=$ $230 \mathrm{~nm}$ is the width of the undoped region of the diode]. The inset in Fig. 4(b) shows the $P_{\text {up }}$ reverse bias dependence for a $p$-type Schottky diode. In general, both $P_{\text {up }}$ and $P_{\text {down }}$ increase with $B$ and reverse bias, but also the difference between the two thresholds increases, leading to a broader range of incident powers in which the bistability occurs. The threshold bias dependence arises from the influence of the electric field on the carrier population and hence on the electron spin dynamics in the dot [15], the latter also influenced by the interaction with the continuum of states in the contact [16]. These effects will be discussed in detail elsewhere.

In order to explain the nuclear spin switching and bistability, we employ a model based on spin-flip assisted $e-h$ recombination $[8,10]$. We assume that the electron spin is defined by the sign of the circularly polarized excitation $\left(\sigma^{ \pm}\right)$, whereas the hole spin is partially randomized during the energy relaxation. Thus, dark and bright excitons can be formed in the dot ground state, with the rates $\alpha w_{x}$ and $(1-\alpha) w_{x}$, respectively. A bright exciton recombines with the rate $w_{\text {rec }}$ without spin transfer to the nuclei. In contrast, a dark exciton can recombine with the electron simulta- neously flipping its spin due to the hyperfine interaction: the electron virtually occupies an optically active state with the opposite spin and the same energy $[8,10]$ transferring spin to nuclei and, then recombines with the hole with the rate $w_{\text {rec }} N p_{\mathrm{hf}}$, where $p_{\mathrm{hf}}$ depends on the electron Zeeman splitting, $E_{\mathrm{eZ}}=\left|g_{e}\right| \mu_{B}\left[B \pm B_{N}\left(\sigma^{ \pm}\right)\right]$. For the case of $\sigma^{-}$ excitation, polarization of the nuclei leads to a decrease of $E_{\mathrm{eZ}}$, and thus a positive feedback and speeding up of the spin transfer process: the more spin is pumped into the nuclear system the faster becomes the spin transfer rate. By contrast, for $\sigma^{+}$excitation spin transfer leads to an increase of $E_{\mathrm{eZ}}$, leading to the saturation of $S$ (and $B_{N}$ ) at high power.

The spin transfer to the nuclei at a rate $w_{s} \propto \alpha w_{x} N p_{\text {hf }}$ competes with nuclear depolarization, $\dot{S}=-w_{\text {dep }} S$ [see Fig. 1(b)] due to spin diffusion away from the dot into the surrounding GaAs, at a rate $w_{\text {dep }} \sim 1-10 \mathrm{~s}^{-1}[17,18]$. At high power of $\sigma^{-}$excitation $w_{s}$ may exceed $w_{\text {dep }}$, and then a stimulated nuclear polarization will take place due to the positive feedback mechanism described above leading to an abrupt increase of the nuclear spin (at $P=P_{\text {up }}$ ). To achieve the condition $w_{s}=w_{\text {dep }}$ a higher $w_{x}$ (power) will be required at higher $B$ in agreement with the observation in the inset of Fig. 3. The stimulation at $P \approx P_{\text {up }}$ stops when either (i) $\left|E_{\text {eZ }}\right|$ starts increasing again since $B_{N}>B$, causing reduction of $w_{s}$ or (ii) the maximum achievable $B_{N}=B_{N}^{\max }$ in the given dot is reached. This explains the dependence in Fig. 3, where $\Delta E_{N}$, and hence the nuclear field, increases at low $B$ and saturates at high fields, from which we estimate $B_{N}^{\max } \approx 2.5-3 \mathrm{~T}$ [19].

When the power is reduced from beyond the threshold $P_{\text {up }}$ and the condition $w_{s}<w_{\text {dep }}$ is reached at sufficiently low $w_{x}$, a strong negative feedback is expected: further nuclear depolarization will lead to even lower $w_{s}$ due to the increase in the electron Zeeman energy $E_{\mathrm{eZ}}$. Thus, an abrupt nuclear depolarization will take place at a threshold power $P_{\text {down }}<P_{\text {up }}$. This explains the hysteresis behavior in Fig. 4 which accounts for the existence of a bistable nuclear polarization state for $P_{\text {down }}<P<P_{\text {up }}$.

To model this bistability, we solve the rate equations for the nuclear spin polarization $S$, and populations of bright and dark excitons, $n_{b}$ and $n_{d}$,

$$
\begin{aligned}
\dot{S} & =n_{d} w_{\mathrm{rec}} p_{\mathrm{hf}}(1-S)-w_{\mathrm{dep}} S, \\
\dot{n}_{b} & =(1-\alpha) w_{x}\left[1-n_{b}-n_{d}\right]-w_{\mathrm{rec}} n_{b} ; \\
\dot{n}_{d} & =\alpha w_{x}\left[1-n_{b}-n_{d}\right]-\frac{1}{2}(1-S) N w_{\mathrm{rec}} p_{\mathrm{hf}} n_{d},
\end{aligned}
$$

where $1-n_{b}-n_{d}$ is the probability that the dot is empty, and $p_{\mathrm{hf}}$ is given by Eq. (1). In the limit $\gamma \ll\left|g_{e}\right| \mu_{B} B_{N}^{\max }$ we obtain the following equation for a steady state polarization, $S$, induced by the $\sigma^{ \pm}$excitation:

$$
f(S, b) \equiv S\left[1+b \frac{(x \pm S)^{2}}{1-S}\right]=a
$$


where $x=B / B_{N}^{\max }$, and for $w_{x} \ll w_{\text {rec }}$ (low occupancy of the dot) both $a$ and $b$ are proportional to the excitation power: $a=2 \alpha w_{x} / N w_{\text {dep }}$ and $b=2 \alpha N w_{x} / w_{\text {rec }}$.

For a low excitation power, such that $b \ll 1$, Eq. (2) has a single solution $S \approx a$ for both $\sigma^{+}$and $\sigma^{-}$excitation. In the $\sigma^{+}$excitation case, $f(S, b)$ is a monotonic function and for all $a$ and $b$ a single solution to Eq. (2) is obtained. On the other hand, for $\sigma^{-}$excitation, for higher powers such that $b \gtrsim 1, f(S, b)$ acquires an $N$ shape, as illustrated in Fig. 4(c). As shown in the diagram, an abrupt transition to $S>x(S \approx a)$ will be obtained when $a_{\max }\left(a_{\min }\right)$ is reached at the local maximum (minimum) of $f(S, b)$. The transitions at $a_{\max }$ and $a_{\min }$ correspond to the $P_{\text {up }}$ and $P_{\text {down }}$ thresholds in Fig. 4, respectively, whereas for $a_{\min }<a<$ $a_{\max }$, the polarization degree $S$ enters a regime of bistability in which Eq. (2) has three solutions, two of which are stable with an unstable one in between.

We find [using Eq. (2)] that the occurrence of the switch to $S>x$ depends on the dimensionless ratio $\theta=a / b=$ $w_{\text {rec }} / N^{2} w_{\text {dep }}$, since at small $\theta, a$ will grow more slowly with $w_{x}$ than the magnitude of $f(S, b)$ at the local maximum. $\theta$ is determined by the dot parameters only, and can be estimated for the dots studied in our experiment: we obtain $\theta_{\text {exp }} \sim 1-10$ from $w_{\text {rec }} \sim 10^{9} \mathrm{sec}^{-1}, \quad w_{\text {dep }} \sim$ $1-10 \mathrm{sec}^{-1}$, and $N \sim 10^{4}$. Using Eq. (2) we find that for $x \leq 0.8$ the spin switch is possible for any $\theta_{\exp }>\theta_{c}$, where $\theta_{c}=\frac{1}{16}(3-\sqrt{9-8 x})(4 x-3+\sqrt{9-8 x})^{2} /(1+$ $\sqrt{9-8 x}) \leq 0.1$, which is consistent with our observations. A hysteresis loop calculated using Eq. (2) for $x=0.7$ (with $\theta_{c} \approx 0.07$ ) and $\theta=0.1$ (close to the critical $\theta_{c}$ ), is shown in Fig. 4(d).

To summarize, we have observed a strong optically induced bistability of the nuclear spin polarization in self-assembled InGaAs QDs. We show that nuclear magnetic fields up to $3 \mathrm{~T}$ can be switched on and off in individual dots by varying one of three external controlling parameters: electric and magnetic fields and intensity of circularly polarized excitation. The nuclear spin switch has been observed for InGaAs/GaAs QDs at temperatures $T=$ $15-30 \mathrm{~K}$ and external magnetic fields $B=1-3 \mathrm{~T}$. The effect arises due to the strong feedback of the nuclear spin polarization on the dynamics of the electron-nuclear spin transfer accompanying the radiative recombination process, which is accelerated when the Overhauser and external magnetic fields cancel each other.

We thank R. Oulton, A. Imamoglu, and C. Marcus for discussions. This work has been supported by the Sheffield EPSRC Programme Grant No. GR/S76076, the Lancaster-
EPSRC Portfolio Partnership No. EP/C511743, the EPSRC IRC for Quantum Information Processing, ESFEPSRC network No. EP/D062918, and by the Royal Society. A. I. T. was supported by the EPSRC Advanced Research Grant No. EP/C54563X/1 and research Grant No. EP/C545648/1.

Note added. - Another report on similar nuclear effects has been published recently [20].

[1] A. W. Overhauser, Phys. Rev. 92, 411 (1953).

[2] D. Gammon et al., Science 277, 85 (1997); A. S. Bracker et al., Phys. Rev. Lett. 94, 047402 (2005).

[3] J.R. Petta et al., Science 309, 2180 (2005); F. H.L. Koppens et al., Science 309, 1346 (2005).

[4] P.-F. Braun et al., Phys. Rev. Lett. 94, 116601 (2005).

[5] I. A. Akimov, D. H. Feng, and F. Henneberger, Phys. Rev. Lett. 97, 056602 (2006).

[6] C. W. Lai et al., Phys. Rev. Lett. 96, 167403 (2006).

[7] B. Eble et al., Phys. Rev. B 74, 081306(R) (2006).

[8] S. I. Erlingsson, Y. V. Nazarov, and V.I. Fal'ko, Phys. Rev. B 64, 195306 (2001).

[9] I. A. Merkulov, Al. L. Efros, and M. Rosen, Phys. Rev. B 65, 205309 (2002).

[10] D. Gammon et al., Phys. Rev. Lett. 86, 5176 (2001).

[11] Both $n$ - and $p$-type Schottky devices with a $25 \mathrm{~nm}$ tunnel barrier between the back contact and the dots were employed similar to those reported in R. J. Warburton et al., Nature (London) 405, 926 (2000); R. Oulton et al., Phys. Rev. B 66, 045313 (2002).

[12] During the measurement of the power dependences, the power was varied keeping the sample under constant illumination.

[13] The nuclear spin switch occurs at typical powers incident on the aperture $\approx 1 \mathrm{~mW}$, corresponding in most cases to the dot occupancy below the bi-exciton formation.

[14] The hyperfine interaction is negligible for holes due to the $p$-like nature of the Bloch functions.

[15] S. Laurent et al., Phys. Rev. Lett. 94, 147401 (2005); A. Ebbens et al., Phys. Rev. B 72, 073307 (2005).

[16] J. M. Smith et al., Phys. Rev. Lett. 94, 197402 (2005).

[17] D. Paget, Phys. Rev. B 25, 4444 (1982).

[18] For a dot with the radius $r \sim 5 \mathrm{~nm}$ we estimated $w_{\text {dep }} \sim$ $D_{N} / r^{2} \sim \mu_{n}^{2} /\left(\hbar a r^{2}\right) \sim 1-10 \mathrm{~s}^{-1}$, where $D_{N} \sim \mu_{n}^{2} / \hbar a$ is the coefficient of polarization diffusion due to the dipoledipole interaction $\left(\sim \mu_{n}^{2} / a^{3}\right)$ between magnetic moments $\mu_{n}$ of neighboring nuclei and $a=0.56 \mathrm{~nm}$-lattice constant.

[19] A close value of $B_{N}$ was deduced from the dependence of $\Delta E_{N}(B)$ using $\left|g_{e}\right| \approx 0.5$.

[20] P.-F. Braun et al., Phys. Rev. B 74, 245306 (2006). 